\title{
Increased expression of FERM domain-containing 4A protein is closely associated with the development of rectal cancer
}

\author{
YONGTIAN FAN, DECHUAN LI, JUN QIAN, YONG LIU, HAIYANG FENG and DECHUAN LI \\ Department of Colorectal Surgery, Zhejiang Cancer Hospital, Hangzhou, Zhejiang 310022, P.R. China
}

Received October 11, 2014; Accepted November 3, 2015

DOI: 10.3892/etm.2015.2933

\begin{abstract}
The aim of the present study was to detect the expression levels of FERM domain-containing 4A (FRMD4A) in rectal cancer tissues and peripheral blood and to investigate the correlation between FRMD4A and cancer development. A total of 78 consecutive patients were enrolled in this study. Thirty healthy individuals were used as the control group. The expression of FRMD4A in rectal cancer and the corresponding normal adjacent tissues was detected by immunohistochemistry and western blotting. The expression of FRMD4A mRNA in peripheral blood was detected by reverse transcription-quantitative polymerase chain reaction. The expression of FRMD4A in rectal cancer tissues was found to be negatively correlated with the degree of differentiation, depth of invasion and Dukes' stage. A negative correlation was identified between FRMD4A and epithelial cadherin expression. The expression of FRMD4A in the peripheral blood of patients with rectal cancer was significantly increased compared with that in the control group $(\mathrm{P}<0.05)$. Expression of FRMD4A in the peripheral blood in the patients with lymph node metastasis was significantly increased compared with that in the patients without lymph node metastasis $(\mathrm{P}<0.05)$. These results indicate that the expression of FRMD4A is significantly increased in rectal cancer tissues and the peripheral blood of patients with rectal cancer, and the expression levels of FRMD4A are closely associated with differentiation, invasion of rectal cancer and Dukes' stage. In conclusion, the findings of the present study suggest that FRMD4A may be used as a target for the diagnosis and treatment of rectal cancer.
\end{abstract}

\section{Introduction}

Rectal cancer is a commonly occurring gastrointestinal cancer, which has a high mortality rate of $\sim 50 \%$ (1). Successful early

Correspondence to: Dr Dechuan Li, Department of Colorectal Surgery, Zhejiang Cancer Hospital, 38 Guangji Road, Hangzhou, Zhejiang 310022, P.R. China

E-mail: 1dcsyyc@163.com

Key words: FERM domain-containing 4A, rectal cancer, western blotting, immunohistochemistry detection of rectal cancer is rare, resulting in a poor prognosis (2). The incidence of rectal cancer is increasing year by year (3), and poses a serious threat to human health. Therefore, it is important to identify novel tumor markers for the early diagnosis and prognosis of rectal cancer.

FERM domain-containing 4A (FRMD4A), located on human chromosome 10 , is a cancer stem cell marker that belongs to the FERM superfamily, all the members of which contain a FERM domain (4). The FERM domain has been demonstrated to play an important role in the cytoskeleton, the maintenance of cell morphology, protein localization and tumor cell migration (5-10). Levels of FRMD4A have been found to be upregulated in skin cancer and squamous cell carcinoma, and are closely associated with recurrence and metastasis (11). Genome-wide association analyses have shown that FRMD4A is highly associated with drug dependence $(12,13)$. FERMD4A regulates the expression of cytoskeletal proteins and the connections between mammary epithelial cells through the activation of ADP ribosylation factor 6 (14). However, the expression of FRMD4A in rectal cancer remains unclear.

In this study, immunohistochemistry, western blotting and reverse transcription-quantitative polymerase chain reaction (RT-qPCR) experiments were performed to detect the expression of FRMD4A in rectal cancer. The correlation between FRMD4A and cancer development was then studied, with the aim of providing experimental evidence concerning the functions of FRMD4A in rectal cancer.

\section{Materials and methods}

Patients. A total of 78 consecutive patients diagnosed with rectal cancer at Zhejiang Cancer Hospital (Zhejiang, China) between September 2013 and December 2014 were enrolled in this study. The study was approved by the Ethics Review Board of Zhejiang Cancer Hospital (Hangzhou, China). Prior written and informed consent was obtained from every patient. General information about the patients is shown in Table I. Fresh rectal cancer and corresponding normal adjacent tissues (distance from tumor edge, $\geq 5 \mathrm{~cm}$ ) were collected, and stored at $-80^{\circ} \mathrm{C}$. The average age of these patients was 53.70 years, with an age range of 32-71 years. There were 42 male patients and 36 female patients. Thirty healthy individuals were used as the control group. The patients underwent categorization for lymph node metastases 
as follows: N0 group, no evidence of lymph node metastasis; N1 group, metastasis to lymph node.

Reagents. Rabbit anti-human FRMD4A polyclonal antibody (ab122475), rabbit anti-human epithelial cadherin (E-cadherin) polyclonal antibody (ab15148) and mouse anti-human glyceraldehyde-3-phosphate dehydrogenase (GAPDH) monoclonal antibody (ab8245) were all purchased from Abcam (Cambridge, UK). Secondary antibodies horseradish peroxidase (HRP)-conjugated goat anti-mouse IgG (ab6789) and HRP-conjugated goat anti-rabbit IgG (ab6721) were also purchased from Abcam. An EnVision Immunohistochemistry Detection kit was purchased from Dako (Glostrup, Denmark). Easyspin whole blood RNA rapid extraction kit (centrifugal column) was purchased from Biomed (Beijing, China). A reverse transcription kit was purchased from Boruike Biotech (Chengdu, China). SYBR Green Real-Time PCR reagents were purchased from Kapa Biosystems (Wilmington, MA, USA).

$R T$-qPCR. Total RNA was extracted from peripheral blood samples, using the whole blood RNA extraction kit according to the manufacturer's protocol. The quality of the RNA was detected by electrophoresis and the spectrophotometric determination of the ratio of optical density at 260 and $280 \mathrm{~nm}$. The RNA was reverse transcribed into cDNA using the reverse transcription kit according to the manufacturer's protocol. qPCR was then conducted using an ABI StepOne Plus Real-Time PCR system (Thermo Fisher Scientific, Foster City, CA, USA). GAPDH was used as an internal control. The relative amount of FRMD4A transcript was expressed as a ratio relative to the level of GAPDH mRNA. The experiments were repeated independently $\geq 3$ times. The primers for FRMD4A were 5'-GATTCTTCGGATGCGTAA-3' and 5'-CTGGCT CACAACATAGTC-3'. The primers for GAPDH were 5'-ATG CTGGCGCTGAGTACGTC-3' and 5'-GGTCATGAGTCC TTCCACGATA-3'.

Immunohistochemistry. Rectal cancer tissues were fixed with $4 \%$ neutral formalin, followed by embedding in paraffin. Then $4-\mu \mathrm{m}$ serial sections were treated with citrate buffer for antigen retrieval at $95^{\circ} \mathrm{C}$ for $10 \mathrm{~min}$. The primary rabbit anti-human FRMD4A polyclonal antibody or rabbit anti-human E-cadherin polyclonal antibody (dilution, 1:200) was added and the sections were incubated for $60 \mathrm{~min}$ at $37^{\circ} \mathrm{C}$. The solution from the EnVision kit was then added and the sections were incubated for $30 \mathrm{~min}$ at $37^{\circ} \mathrm{C}$. The sections were developed with diaminobenzidine chromogenic reagent and observed under a microscope (Olympus BX51; Olympus Corp., Tokyo, Japan). Cells with brown yellow staining were defined as FRMD4A-positive or E-cadherin-positive cells. Ten fields at high magnification (x200) were randomly taken from each section. The intensity of staining and the proportion of positive cells were determined, and each was scored on a scale of 0-3. For staining intensity: Score 0, no staining; score 1, yellow staining; score 2, brown yellow staining; and score 3 , brown staining. For the positive rate: Score 0, 0\% positive cells; score $1,<10 \%$ positive cells; score $2,10-50 \%$ positive cells; and score $3,>50 \%$ positive cells. The FRMD4A and E-cadherin staining indices were calculated by multiplying the score for the intensity of staining by the score for the proportion of positive cells, and graded as follows: Grade 1, score 0-3; grade 2, score 4-6; and grade 3, score 7-9. At least 200 cells were counted in each field.

Western blotting. Total proteins were harvested from $100 \mathrm{mg}$ rectal cancer tissues and separated on $10 \%$ sodium dodecyl sulfate-polyacrylamide gel electrophoresis gels. Then, the proteins were transferred to a polyvinylidene difluoride (PVDF) membrane (Thermo Fisher Scientific, Inc, Vilnius, Lithuania). After blocking with 5\% non-fat milk, the primary antibodies against FRMD4A (dilution, 1:800) and GAPDH (dilution, 1:4,000) were added and the membrane was incubated overnight at $4^{\circ} \mathrm{C}$. The secondary HRP-conjugated $\mathrm{IgG}$ antibodies (goat anti-mouse, 1:5,000; goat anti-rabbit, 1:2,000) were added and the samples were incubated for $1 \mathrm{~h}$ at room temperature. The bound antibodies were detected using an enhanced chemiluminescence system (Beyotime Institute of Biotechnology, Jiangsu, China). The protein expression levels were analyzed using Quantity One software, version 4.6.2 (Bio-Rad Laboratories, Hercules, CA, USA)

Statistical analysis. Statistical analysis software (SPSS, version 10.0; SPSS, Inc., Chicago, IL, USA) was used to conduct $\chi^{2}$ tests. Spearman's correlation was used to test the association between two variables. $\mathrm{P}<0.05$ was considered to indicate a statistically significant result.

\section{Results}

Expression of FRMD4A is upregulated in rectal cancer tissues. To evaluate the expression of FRMD4A in rectal cancer, immunohistochemical analysis of tissue samples taken from patients was performed. Representative results are shown in Fig. 1. Cells with brown and/or yellow staining were FRMD4A-positive. The FRMD4A-positive rate in the rectal cancer tissues was significantly higher than that in the normal adjacent tissues ( 87.0 vs. $25.3 \%$; $\mathrm{P}<0.05)$. These results indicate that the expression level of FRMD4A is increased in rectal cancer.

Correlation between the expression of FRMD4A and clinicopathological features in rectal cancer. To determine the correlation between the expression of FRMD4A in rectal cancer and clinicopathological features, Spearman correlation analysis was performed. As shown in Table I, there were no statistically significant differences in FRMD4A expression among the patients according to age, gender or tumor size $(\mathrm{P}>0.05)$. However, the expression of FRMD4A was found to be negatively correlated with the degree of differentiation, depth of invasion, Dukes' stage and lymph node metastasis (Table I). These results indicate that positive correlations exist between FRMD4A and certain clinicopathological features of patients with rectal cancer, namely the degree of differentiation, depth of invasion, Dukes' stage and lymph node metastasis.

Negative correlation between FRMD4A and E-cadherin in rectal cancer patients. To determine the correlation between FRMD4A and E-cadherin in rectal cancer, 
Table I. Correlation between FRMD4A expression in rectal cancer patients and cancer development.

\begin{tabular}{|c|c|c|c|c|c|c|c|c|}
\hline \multirow[b]{2}{*}{ Characteristic } & \multirow[b]{2}{*}{ Cases $(n=78)$} & \multicolumn{3}{|c|}{$\begin{array}{l}\text { Immunohistochemical } \\
\text { scoring index }\end{array}$} & \multicolumn{2}{|c|}{$\chi^{2}$} & \multicolumn{2}{|c|}{ Correlation } \\
\hline & & $0-3(n=16)$ & $4-6(n=49)$ & $9(n=13)$ & $\chi^{2}$-value & P-value & Pearson's r & P-value \\
\hline Age (years) & & & & & 1.354 & 0.852 & & \\
\hline$<40$ & 5 & 2 & 2 & 1 & & & & \\
\hline $41-65$ & 43 & 8 & 28 & 7 & & & & \\
\hline$>66$ & 30 & 6 & 19 & 5 & & & & \\
\hline Gender & & & & & 2.385 & 0.184 & & \\
\hline Male & 42 & 9 & 29 & 4 & & & & \\
\hline Female & 36 & 7 & 20 & 9 & & & & \\
\hline Tumor size & & & & & 0.111 & 0.946 & & \\
\hline$<5 \mathrm{~cm}$ & 26 & 5 & 17 & 4 & & & & \\
\hline$\geq 5 \mathrm{~cm}$ & 52 & 11 & 32 & 9 & & & & \\
\hline Differentiation status & & & & & 18.902 & 0.001 & 0.339 & 0.002 \\
\hline High & 16 & 9 & 7 & 0 & & & & \\
\hline Moderate & 33 & 5 & 20 & 8 & & & & \\
\hline Poor & 29 & 2 & 22 & 5 & & & & \\
\hline \multicolumn{9}{|l|}{ Depth of invasion } \\
\hline $\mathrm{T} 2$ & 5 & 2 & 3 & 0 & 11.128 & 0.025 & 0.352 & 0.002 \\
\hline $\mathrm{T} 3$ & 56 & 13 & 37 & 6 & & & & \\
\hline $\mathrm{T} 4$ & 17 & 1 & 9 & 7 & & & & \\
\hline \multicolumn{9}{|l|}{ Dukes' stage } \\
\hline $\mathrm{B}$ & 46 & 12 & 30 & 4 & 10.805 & 0.029 & 0.308 & 0.006 \\
\hline $\mathrm{C}$ & 25 & 4 & 16 & 5 & & & & \\
\hline $\mathrm{D}$ & 7 & 0 & 3 & 4 & & & & \\
\hline \multicolumn{9}{|l|}{ Lymph node metastasis } \\
\hline N0 & 30 & 11 & 15 & 4 & 7.802 & 0.020 & 0.257 & 0.023 \\
\hline N1 & 48 & 5 & 34 & 9 & & & & \\
\hline
\end{tabular}

FRMD4A, FERM domain-containing 4A; N0, no evidence of lymph node metastasis; N1, metastasis to lymph node.
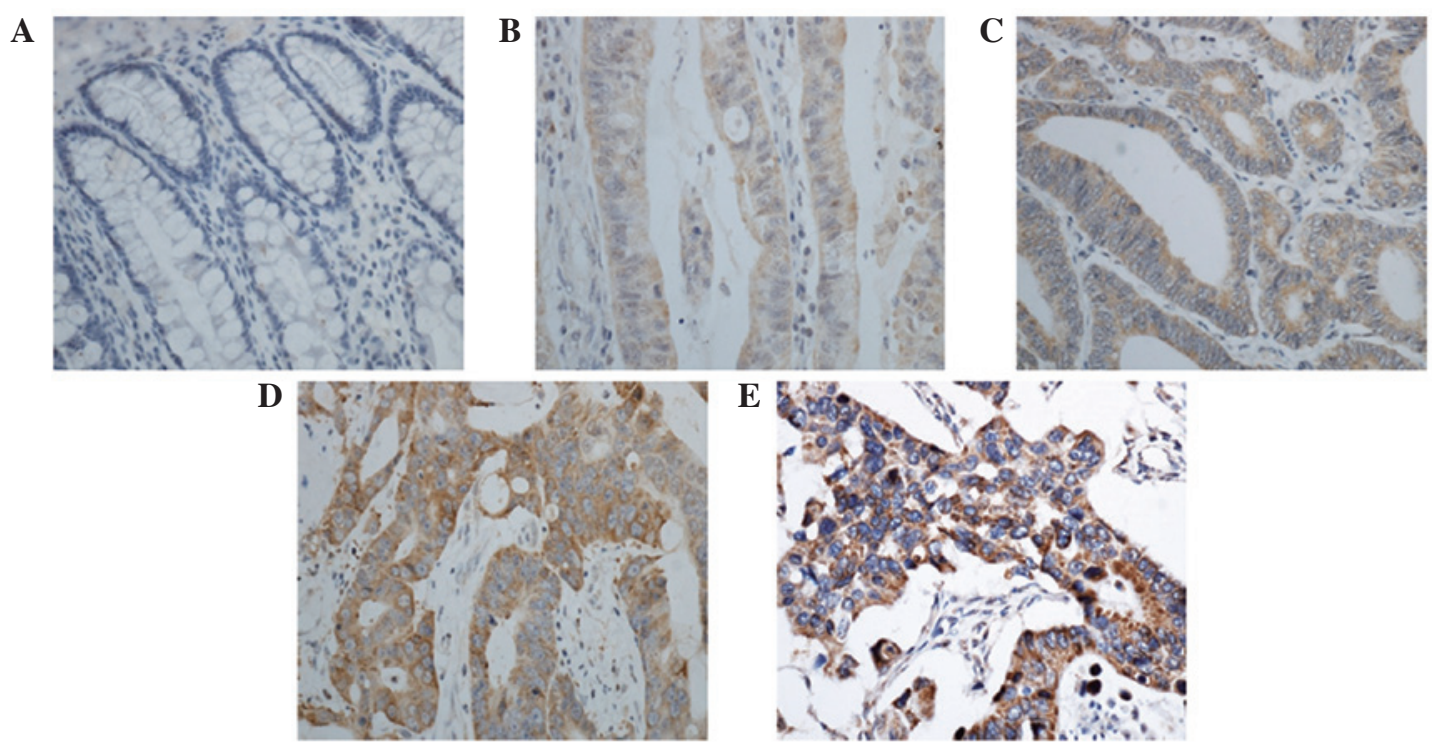

Figure 1. Expression of FRMD4A in rectal cancer tissues. The EnVision immunohistochemical method was performed to detect the expression of FRMD4A in the rectal cancer tissues. Cells with brown and/or yellow staining were defined as FRMD4A-positive. Experiments were repeated at least 3 times. Expression of FRMD4A in the (A) normal tissues adjacent to rectal cancer, (B) rectal cancer tissues, (C) well-differentiated rectal cancer tissues, (D) moderately differentiated rectal cancer tissues and (E) poorly differentiated rectal cancer tissues (magnification, x200). FRMD4A, FERM domain-containing 4A. 
A

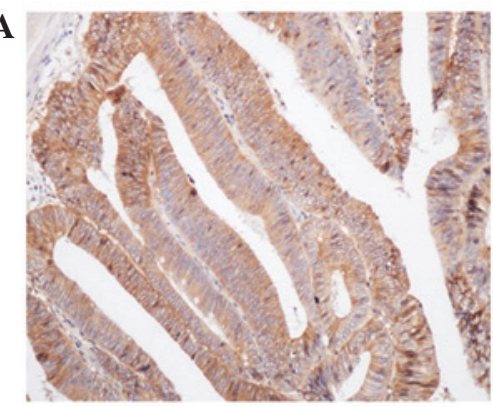

B

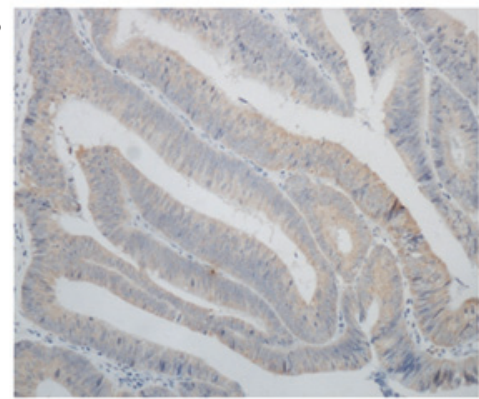

Figure 2. Correlation between FRMD4A and E-cadherin expression in rectal cancer tissues. The EnVision immunohistochemical method was performed to detect the expression of FRMD4A and E-cadherin in the rectal cancer tissues. Spearman's correlation analysis was performed to determine the correlation between FRMD4A and E-cadherin. Experiments were repeated at least 3 times. Expression of (A) FRMD4A and (B) E-cadherin in the rectal cancer tissues (magnification, x200). FRMD4A, FERM domain-containing 4A.

A

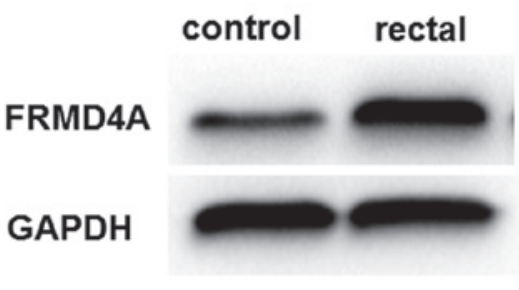

C

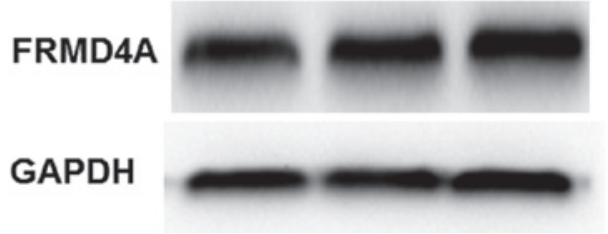

B

FRMD4A

GAPDH

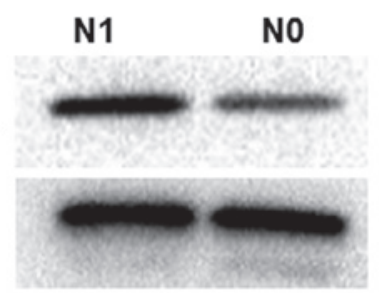

Dukes' A/B Dukes' C/D

FRMD4A

GAPDH

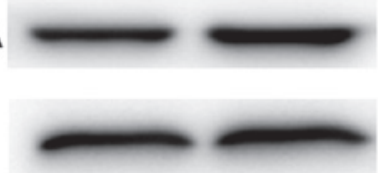

Figure 3. Expression of FRMD4A protein in rectal cancer tissues. Total proteins were harvested from the tumor samples and controls and analyzed by western blotting to detect the expression of FRMD4A protein. GAPDH was used as a loading control. The experiments were repeated at least 3 times. Representative blots of FRMD4A protein in the (A) rectal cancer patients and the normal adjacent tissues, (B) the N0 and N1 groups, (C) highly, moderately and poorly differentiated rectal cancer tissues and (D) the Dukes' stage A and B and Dukes' stage C and D groups. FRMD4A, FERM domain-containing 4A; GAPDH, glyceraldehyde 3-phosphate dehydrogenase; N0, no lymph node metastasis; N1, metastasis to lymph node.

immunohistochemical analysis was used to detect the expression of the two proteins in rectal cancer tissue samples (Fig. 2). Spearman correlation analysis was performed to analyze the correlation between FRMD4A and E-cadherin expression in rectal cancer. In the tissue sections, FRMD4A expression was found to be negatively correlated with E-cadherin expression ( $\mathrm{r}=-0.410, \mathrm{P}<0.01)$, indicating that a negative correlation exists between these two proteins in rectal cancer.

Expression of FRMD4A protein is increased in rectal cancer. To determine the expression of FRMD4A protein in rectal cancer, western blotting was performed. As shown in Fig. 3, the relative expression level of FRMD4A protein in rectal cancer tissues $(3.97 \pm 0.20)$ was significantly increased when compared with that in the normal adjacent tissues $(\mathrm{P}<0.05)$. The relative expression level of FRMD4A protein in the N1 group (2.76 \pm 0.31$)$ was significantly increased when compared with that in the N0 group (normalized value $1 ; \mathrm{P}<0.05$ ). The expression level of FRMD4A protein in cancers of Dukes' stage $C$ and D $(1.78 \pm 0.24)$ was significantly higher compared with that in cancers of Dukes' stages A and B (normalized value $1 ; \mathrm{P}<0.05)$. The correlation between the FRMD4A expression level and the degree of differentiation of the rectal cancer was observed to be negative. These results indicate that the expression of FRMD4A protein is increased in rectal cancer tissue. FRMD4A may be associated with the invasion and metastasis of rectal cancer, and negatively correlates with the degree of differentiation.

Expression of FRMD4A mRNA is increased in the peripheral blood of rectal cancer patients. To determine the expression of FRMD4A mRNA in the peripheral blood of rectal cancer patients, RT-qPCR analysis was performed. As shown in Fig. 4, the expression of FRMD4A mRNA in the peripheral blood of patients with rectal cancer $(3.24 \pm 0.13)$ was significantly increased compared with that in healthy individuals $(\mathrm{P}<0.05)$. The expression of FRMD4A mRNA in the N1 group $(4.29 \pm 0.20)$ was significantly increased compared with that in the N0 group $(2.31 \pm 0.28)$ and the control $(\mathrm{P}<0.05)$. Expression levels of FRMD4A mRNA in cancers of Dukes' stages $\mathrm{C}$ and $\mathrm{D}$ were significantly increased compared with those in cancers of Dukes' stages A and B $(\mathrm{P}<0.05)$. These 
A

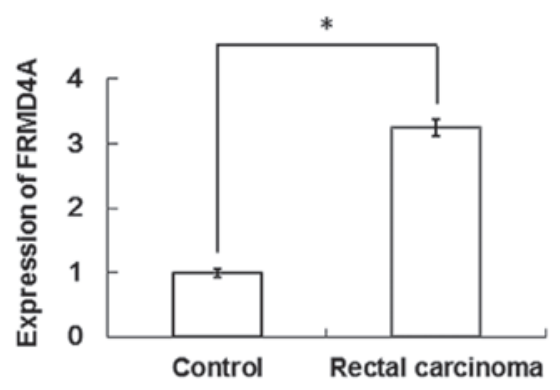

B

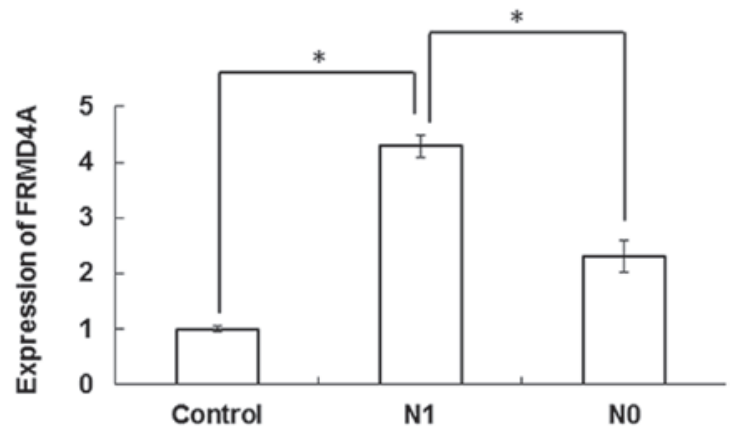

C

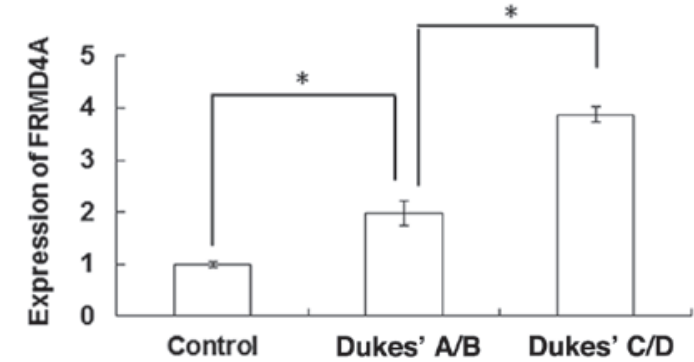

Figure 4. RT-qPCR detection of FRMD4A mRNA. Total RNA was harvested from tissues and RT-qPCR was performed to analyze the FRMD4A mRNA levels in rectal cancer patients. The levels (mean value) of FRMD4A transcript were calculated. Error bars show standard deviation. The experiments were repeated at least 3 times. Expression of FRMD4A mRNA in (A) rectal cancer and control, (B) the N0 and N1 groups and (C) the Dukes' stage A and B and Dukes' stage C and D groups. " $\mathrm{P}<0.05$. RT-qPCR, reverse transcription-quantitative polymerase chain reaction; FRMD4A, FERM domain-containing 4A; N0, no lymph node metastasis; N1, metastasis to lymph node.

results indicate that the expression of FRMD4A mRNA in peripheral blood is increased in patients with rectal cancer. The expression of FRMD4A mRNA was increased as the Dukes' stage of the cancer increased.

\section{Discussion}

It has been reported that the expression of FRMD4A is changed in some tumors and has an association with tumor invasion and metastasis $(11,15)$. For example, Goldie et al found that FRMD4A expression levels are increased in squamous cell carcinoma, and demonstrated that FRMD4A-knockdown reduces tumor growth and metastasis (11). In the present study, immunohistochemistry and western blotting assays were performed to detect the expression of FRMD4A in rectal cancer tissue. The results showed that the expression of FRMD4A in rectal cancer tissue was significantly increased when compared with that in the normal adjacent tissues $(\mathrm{P}<0.05)$. Positive staining of FRMD4A protein was observed mainly in the cytoplasm, and partly in nucleus. The level of expression of FRMD4A increased as the Dukes' stage and the degree of differentiation decreased in the rectal cancer. The expression of FRMD4A in patients with lymph node metastasis was significantly increased compared with that in patients without lymph node metastasis $(\mathrm{P}<0.05)$, indicating that FRMD4A might be closely associated with the development, invasion and metastasis of rectal cancer.

E-cadherin is a marker of epithelial-mesenchymal transition (EMT) and has a close association with tumor invasion and metastasis $(16,17)$. It has been reported that erythrocyte membrane protein band 4.1 like 5 (EPB41L5), which contains the FERM domain, promotes EMT through the regulation of cadherin and integrin expression (18). As mentioned earlier, the FRMD4A protein also contains the FERM domain. Thus, it is possible that FRMD4A is associated with EMT. Therefore, the correlation between FRMD4A and E-cadherin was examined in the present study. Immunohistochemical analysis showed that the expression of FRMD4A was negatively correlated with the expression of E-cadherin in rectal cancer. This suggests that FRMD4A may be involved in EMT and cell adhesion and may promote the invasion and metastasis of rectal cancer. The results of RT-qPCR analysis showed that the expression level of FRMD4A mRNA in the peripheral blood of patients with rectal cancer was significantly increased compared with that in patients in the control group $(\mathrm{P}<0.05)$. The expression levels of FRMD4A mRNA in the peripheral blood of Dukes' stage $C$ and D subgroups were significantly increased compared with those in Dukes' stage A and B subgroups $(\mathrm{P}<0.05)$. Since Dukes' stages and lymph node metastasis are important indicators for the prognosis of patients with rectal cancer (19), increased expression levels of FRMD4A may indicate a poor prognosis for patients with rectal cancer.

In conclusion, the present study indicates that the expression of FRMD4A is closely associated with the development, invasion and metastasis of rectal cancer. Therefore, FRMD4A may play an important role in the early diagnosis and prognosis of patients with rectal cancer. In addition, FRMD4A may be a potential therapeutic target for rectal cancer.

\section{Acknowledgements}

This study was supported by the Natural Science Foundation of Zhejiang Province (grant no. Y207427) and Medicine and 
Health Science Research Fund of Zhejiang Province (grant no. 2008B019).

\section{References}

1. Cui C, Zhang M, Tian L, Jiang W, Zeng Z and Li L: Survival implications of pretreatment pelvic $\mathrm{CT}$ in rectal cancer patients after neoadjuvant chemoradiotherapy and surgery. Int J Clin Exp Med 8: 12801-12809, 2015.

2. Maffione AM, Chondrogiannis S, Capirci C, Galeotti F, Fornasiero A, Crepaldi G, Grassetto G, Rampin L, Marzola MC and Rubello D: Early prediction of response by (18)F-FDG PET/CT during preoperative therapy in locally advanced rectal cancer: A systematic review. Eur J Surg Oncol 40: 1186-1194, 2014.

3. Probst CP, Becerra AZ, Aquina CT, Tejani MA, Hensley BJ, González MG, Noyes K, Monson JR and Fleming FJ: Watch and wait? - Elevated pretreatment CEA is associated with decreased pathological complete response in rectal cancer. J Gastrointest Surg: Nov 6, 2015 (Epub ahead of print).

4. Strizzi L, Hardy KM, Margaryan V, Hillman DW, Seftor EA Chen B, Geiger XJ, Thompson EA, Lingle WL, et al: Potential for the embryonic morphogen Nodal as a prognostic and predictive biomarker in breast cancer. Breast Cancer Res 14: R75, 2012.

5. Murata K, Nunomura W, Takakuwa Y and Cherr GN: Two different unique cardiac isoforms of protein 4.1R in zebrafish, Danio rerio, and insights into their cardiac functions as related to their unique structures. Dev Growth Differ 52: 591-602, 2010

6. Clucas J and Valderrama F: ERM proteins in cancer progression. J Cell Sci 127: 267-275, 2014.

7. Fiévet B, Louvard D and Arpin M: ERM proteins in epithelial cell organization and functions. Biochim Biophys Acta 1773: 653-660, 2007.

8. Tepass U: FERM proteins in animal morphogenesis. Curr Opin Genet Dev 19: 357-367, 2009.

9. Bennett V and Baines AJ: Spectrin and ankyrin-based pathways: Metazoan inventions for integrating cells into tissues. Physiol Rev 81: 1353-1392, 2001
10. Yu H,Zhang Y, Ye L and Jiang WG: The FERM family proteins in cancer invasion and metastasis. Front Biosci (Landmark Ed) 16: 1536-1550, 2011

11. Goldie SJ, Mulder KW, Tan DW, Lyons SK, Sims AH and Watt FM: FRMD4A upregulation in human squamous cell carcinoma promotes tumor growth and metastasis and is associated with poor prognosis. Cancer Res 72: 3424-3436, 2012.

12. Johnson C, Drgon T, Liu QR, Zhang PW, Walther D, Li CY, Anthony JC, Ding Y, Eaton WW and Uhl GR: Genome wide association for substance dependence: Convergent results from epidemiologic and research volunteer samples. BMC Med Genet 9: 113, 2008.

13. Yoon D, Kim YJ, Cui WY, Van der Vaart A, Cho YS, Lee JY, Ma JZ, Payne TJ, Li MD and Park T: Large-scale genome-wide association study of Asian population reveals genetic factors in FRMD4A and other loci influencing smoking initiation and nicotine dependence. Hum Genet 131: 1009-1021, 2012

14. Ikenouchi J and Umeda M: FRMD4A regulates epithelial polarity by connecting Arf6 activation with the PAR complex. Proc Natl Acad Sci USA 107: 748-753, 2010.

15. Zheng XH, Zhao JJ, Jia B, Pan J, Chen J, Qiu XL, Han JS and Chu HX: The influence of inactive FRMD4A gene on the biological behavior of human tongue cancer CAL-27 cell. Shi Yong Yi Xue Za Zhi 10: 99-103, 2014 (In Chinese).

16. Heerboth S, Housman G, Leary M, Longacre M, Byler S, Lapinska K, Willbanks A and Sarkar S: EMT and tumor metastasis. Clin Transl Med 4: 6, 2015.

17. Steinestel K, Eder S, Schrader AJ, and Steinestel J: Clinical significance of epithelial-mesenchymal transition. Clin Transl Med 3: 17, 2014.

18. Hirano M, Hashimoto S, Yonemura S, Sabe H and Aizawa S: EPB41L5 functions to post-transcriptionally regulate cadherin and integrin during epithelial-mesenchymal transition. J Cell Biol 182: 1217-1230, 2008.

19. Radovanović Z, Radovanović D, Breberina M, Petrović T, Golubović A and Bokorov B: The value of endorectal ultrasonography in rectal cancer staging. Med Pregl 61: 557-561, 2008 (In Serbian). 